\title{
Magnetic vortices induced by a moving tip
}

\author{
Martin P. Magiera $^{1}$ (a), Alfred Hucht ${ }^{1}$, Haye Hinrichsen ${ }^{2}$, Silvio R. Dahmen ${ }^{3}$ and Dietrich E. Wolf ${ }^{1}$ \\ 1 Faculty of Physics and CeNIDE, University of Duisburg-Essen, D-47048 Duisburg, Germany, EU \\ 3 Universität Würzburg, Fakultät für Physik und Astronomie, 97074 Würzburg, Germany, EU \\ 2 Instituto de Fisica, Universidade Federal do Rio Grande do Sul, 91501-970 Porto Alegre RS, Brazil
}

PACS 75.70. Kw - Domain structure (including magnetic bubbles and vortices)

PACS 75.78.Fg - Dynamics of domain structures

PACS 75.10.Hk - Classical spin models

\begin{abstract}
A two-dimensional easy-plane ferromagnetic substrate, interacting with a dipolar tip which is magnetised perpendicular with respect to the easy plane is studied numerically by solving the Landau-Lifshitz Gilbert equation. Due to the symmetry of the dipolar field of the tip, in addition to the collinear structure a magnetic vortex structure becomes stable. It is robust against excitations caused by the motion of the tip. We show that for high excitations the system may perform a transition between the two states. The influence of domain walls, which may also induce this transition, is examined.
\end{abstract}

Introduction. - Vortices in magnetic layers have been intensely studied 1 1.7]. They are topological defects, and can be characterised by a chirality (an integer winding number, which is a conserved quantity for topological reasons) and a polarity (the out-of plane magnetisation). They can annihilate by antivortices having a windling number of opposite sign. Vortex-antivortex pairs are important excitations in two dimensional magnetic systems. In open systems one can also excite isolated vortices, when the corresponding antivortex leaves the system at the boundary.

The polarity of a vortex may be treated as a bit, as it possesses two very stable states which can be easily probed e.g. with GMR sensors, as those used in reading heads of magnetic disks. Thus magnetic vortex structures are promising candidates for novel non-volatile storage concepts. An important question in this context is, how to "write" a vortex. To switch the polarization, magnetic field pulses [8,9], alternating magnetic fields [10] or spinpolarised currents 11] have been proposed.

In this letter we present and analyse a new method, by which vortices can be generated or removed. The excitation energy is provided by a moving magnetic tip as used in magnetic force microscopy (MFM). It is known that MFM tips do influence the substrate, which they are supposed to probe - an undesired effect for the microscopy purpose. It has been observed in experiments that domain walls

\footnotetext{
(a) E-mail: martin.magiera@uni-due.de
}

are deformed by the passing tip 12,13. Tip controlled domain wall manipulation has been achieved 14,15. The manipulation of vortices by an MFM tip has been realized in a type-II superconductor 16,17. Thus, it is of great interest from the microscopy and the manipulatory point of view to study how an MFM tip interacts with the scanned surface.

We first show that vortex states are stable configurations in easy plane ferromagnetic structures in the presence of a magnetic tip, positioned above the ferromagnetic structure. Such a system has been studied recently in order to explore the friction force decelerating the magnetic tip 18 20]. Then we show that the vortex remains stable when the tip is moved along the substrate, dragging the vortex through the substrate. Moreover the moving tip may also create or destroy a vortex structure, depending on the tip magnetisation and velocity, offering an alternative way to switch between three states: A collinear state, as well as a vortex state with up or down polarity. Finally we study the stability of the dragged vortex structure when it passes through a domain wall.

The system. - Our system consists of $N=L_{x} \times L_{y}$ classical Heisenberg spins $\mathbf{S}_{i}=\boldsymbol{\mu}_{i} / \mu_{s}$ on a square grid, where $\mu_{s}$ is a material specific saturation magnetisation. The Hamiltonian contains two terms, corresponding to a substrate and a tip part

$$
\mathcal{H}=\mathcal{H}_{\text {sub }}+\mathcal{H}_{\text {tip }},
$$




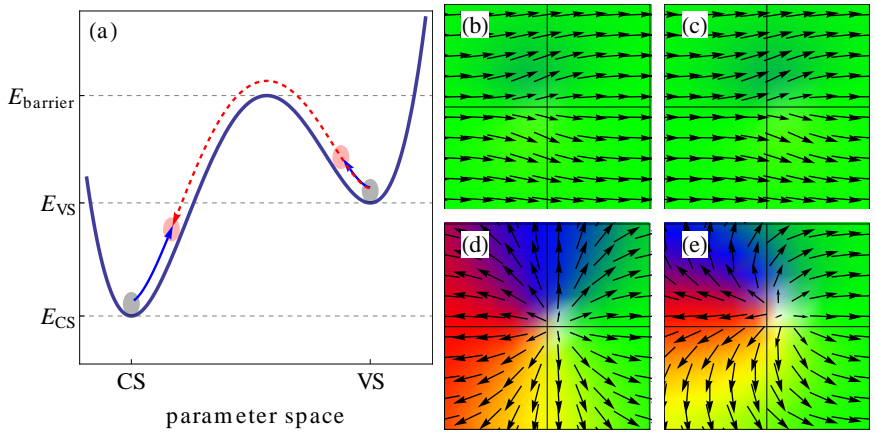

Fig. 1: (Colour on-line) (a) Sketch of the energy landscape. At least two local minima, corresponding to the CS (collinear state) and the VS (vortex state), exist. The absolute minimum depends on the tip strength $w$. There is a potential barrier between the two states and thus the CS and the VS may be very stable against external driving (blue arrows). A strong perturbation may lead to the transition from the VS to the CS (red dashed arrow) and vice versa. (b-e) A part of a system near equilibrium, initialised in the CS $(b, c)$ or the VS $(d, e)$. The scanning velocities are $v=0.01(\mathrm{~b}, \mathrm{~d})$ and $v=0.3(\mathrm{c}, \mathrm{e})$. The colour coding as well as the arrows represent the magnetisation in the $x y$-plane, $c f$. fig. 4

For the spin-spin interaction in the substrate we assume an isotropic exchange with interaction constant $J$ and equivalent easy axes in $x$ - and $y$-direction,

$\mathcal{H}_{\text {sub }}=-J \sum_{\langle i, j\rangle} \mathbf{S}_{i} \cdot \mathbf{S}_{j}-d_{z} \sum_{i} S_{i, z}^{2}-e_{x y} \sum_{i}\left(S_{i, x}^{4}+S_{i, y}^{4}\right)$.

The easy-plane anisotropy $d_{z}<0$ alone would lead to an infinite domain wall width, as all in-plane magnetisation configurations would be degenerated. Correspondingly, the Mermin-Wagner theorem would rule out long range magnetic order at finite temperatures 21]. The fourthorder anisotropy term $e_{x y}>0$, however, breaks the continuous symmetry, so that the Hamiltonian (2) has a ferromagnetic low temperature phase with domain walls of finite width. We use the anisotropy parameters $d_{z}=-0.1 J$ and $e_{x y}=0.1 J$ in this letter.

The substrate-tip interaction is introduced via a dipolar term,

$$
\mathcal{H}_{\mathrm{tip}}=-w \sum_{i} \frac{3\left(\mathbf{S}_{i} \cdot \mathbf{e}_{i}\right)\left(\mathbf{S}_{\mathrm{tip}} \cdot \mathbf{e}_{i}\right)-\mathbf{S}_{i} \cdot \mathbf{S}_{\mathrm{tip}}}{R_{i}^{3}},
$$

where $R_{i}=\left|\mathbf{R}_{i}\right|$ denotes the norm of the position of spin $i$ relative to the tip $\mathbf{R}_{i}=\mathbf{r}_{i}-\mathbf{r}_{\text {tip }}$, and $\mathbf{e}_{i}$ its unit vector $\mathbf{e}_{i}=\mathbf{R}_{i} / R_{i} . \mathbf{r}_{i}$ and $\mathbf{r}_{\text {tip }}$ are the position vectors of the substrate spins and the tip respectively. $w$ is a free parameter that quantifies the dipole-dipole-coupling between the substrate spins and the tip, thus controlling the strength of the tip. We use $\mathbf{S}_{\text {tip }}=(0,0,-1), w$ is a free parameter representing the tip strength. The tip is moved with constant velocity $(v, 0,0)$ two lattice constants above the substrate, along the middle line between two spin rows.
For a real system the tip is of course not a point dipole as represented by (3). It rather resembles a magnetic cone of micron length. Depending on the scanned length scales the distance between surface and probe, as well as on the length and shape of the probe, different approximations of the tip field are used, see Ref. 22 and references therein. While the dipole approximation yields the correct far-field behavior, it has been shown in Refs. 2325 that the stray field of a hollow cone-type tip may be approximated by a magnetic point charge, if the tip extension is large compared to the distance between tip and surface. As the tip magnetization is assumed perpendicular to the surface in this work, both the dipole and the monopole approximation favor a vortex directly underneath the tip. The effects described in the following are therefore expected to be qualitatively similar in both cases, however they may be more pronounced for the monopole field due to its longer range.

Two different kinds of boundary conditions will be used. First we consider the case that all inhomogeneities in the system are due to the tip. Then it is natural to describe the system in the comoving frame of the tip [18]: In the $y$-direction, the boundaries are open, in the $x$-direction dynamical. When the tip advances by exactly one lattice constant, the foremost row is duplicated, and the last one is deleted. That way, arbitrarily long times can be simulated. In the second part we investigate, how vortex generation is influenced by a domain wall that is pinned far away from the tip. In this case, a combination of open ( $x$-direction) and fixed ( $y$-direction) boundary conditions is more appropriate. The system sizes used are $64 \times 48$ for the case of simulations in the absence of domain walls, and $200 \times 48$ for the case where domain walls are of interest.

The equation of motion is the Landau-Lifshitz-Gilbert (LLG) equation 26, 27.

$$
\frac{\partial}{\partial t} \mathbf{S}_{i}=-\frac{\gamma}{\left(1+\alpha^{2}\right) \mu_{s}}\left[\mathbf{S}_{i} \times \mathbf{h}_{i}+\alpha \mathbf{S}_{i} \times\left(\mathbf{S}_{i} \times \mathbf{h}_{i}\right)\right],
$$

with saturation magnetisation $\mu_{s}$, gyromagnetic ratio $\gamma$, the phenomenological damping constant $\alpha$ (we use the high damping value $\alpha=0.5$ in this letter to reach a steady state in a short simulation time) and the local field $\mathbf{h}_{i}=$ $-\partial \mathcal{H} / \partial \mathbf{S}_{i}$. It produces Larmor precession with frequency $\left|\mathbf{h}_{i}\right| \gamma / \mu_{s}$, and a damping in the direction of the local field. In equilibrium, each spin points in the direction of its local field. To solve the LLG we use the Heun integration scheme 28.

Nonequilibrium steady states. - Before studying the driven system, let us discuss the equilibrium case $(v=0)$. If the tip is absent $(w=0)$, the equilibrium configuration corresponds to all spins pointing in the same direction, the collinear state (CS).

As we increase the tip strength, a second minimum appears in the potential landscape, the cylindrically symmetric vortex state (VS, cf. fig. 11(d)). At the same time, 


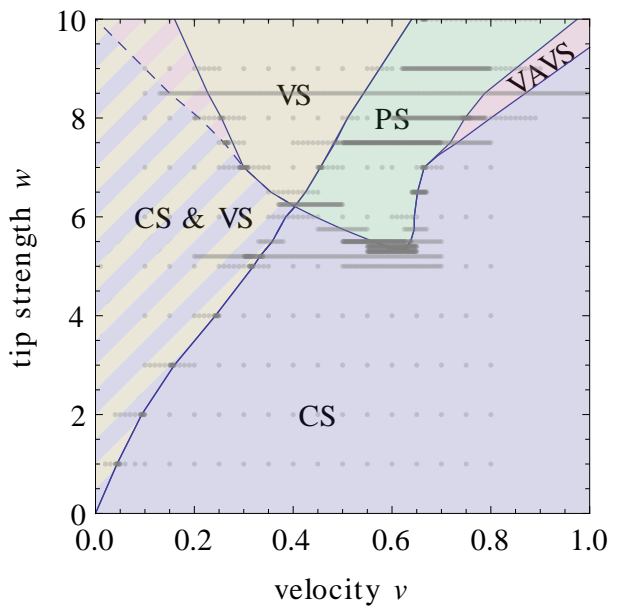

Fig. 2: (Colour on-line) Nonequilibrium steady state diagram with the pure states (collinear state, CS, and vortex state, VS), the co-existence state (CS \& VS), the periodic state (PS) and the vortex-antivortex state (VAVS). The grey points represent the underlying simulations.

the CS minimum is continuously moved to the configuration of a slightly disturbed collinear configuration ( $c f$. fig. 1 (b)). For any reasonably small tip strength, both equilibrium states, the CS and the VS, are stable. If we initialise a VS, and switch off the tip, the VS is still stable: Being a topological defect, its winding number is a conserved quantity which cannot spontaneously change.

Let us now move the tip with constant velocity $(v>0)$ : The system is driven into a nonequilibrium steady state (NESS), which is near the equilibrium for small excitations. Accordingly, in the NESS diagram (cf. fig. 2) we see at $v>0$ a region where the CS and the VS coexist. Here the initial configuration determines the finally stabilised NESS. As the excitation overcomes a threshold by increasing the tip strength $w$ or scanning velocity 21 , the system may perform a transition from the VS to the CS (when the CS represents the total minimum) or vice versa, as sketched in fig. 1(a) by the red dashed arrow. In the NESS, we then observe either the pure CS or the pure VS.

Another mixed state is the periodic state: Here the system is excited so strongly that neither the VS nor the CS is stable. The system then flips between the states back and forth continuously. We explain this state in more detail, because it sheds also more light onto the transitions to the pure states mentioned above. Let us start in the VS. Here the vortex is bound by the tip, because the cylindrically symmetric structure minimises the tip energy. However, in the PS (or when the CS is the corresponding NESS) the tip pumps so much energy into the substrate that the vortex may decouple from the tip, and moves away towards the system boundary (fig. 3(b)), leaving a CS behind (fig. 3(c). Accordingly the potential barrier in fig. 1(a) corre-

\footnotetext{
${ }^{1} \mathrm{~A}$ value $w=1$ in our model corresponds to a magnetic field of about $0.1 \mathrm{~T}$ in the substrate under the tip.
}
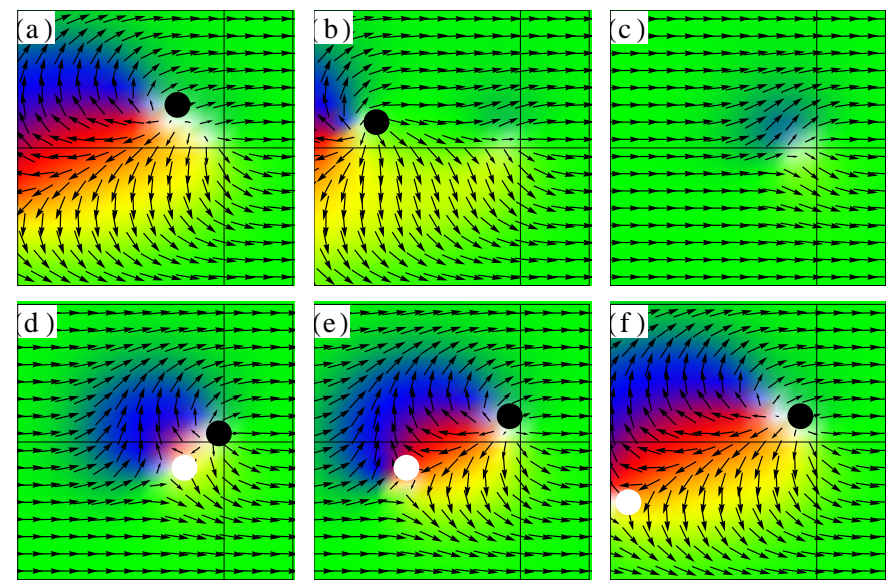

Fig. 3: (As a movie and colour on-line) Some snapshots of a system in the PS in a co-moving frame of reference, the tip positioned at the origin (the two lines correspond to the $x$ and $y$-axis). Vortex cores are marked as black dots, antivortex cores as white dots. Here we observe the periodical transition from the VS to the CS (a-c) via the release of the vortex from the tip, as well as the transition from the CS to the VS (c-f) via a VAVP creation. See the complete cycle as a movie online.

sponds to the energy of a free moving vortex and a CS. If the system's NESS is the CS, the system has now reached its steady state. If the initial state was the CS, but the corresponding NESS is the VS or the PS, the system is again excited further by the tip, and a vortex-antivortex pair (VAVP) may nucleate under the tip (fig. 3(d)). The energy of a VAVP represents the potential barrier which the system has to overcome at this transition. After the nucleation, the antivortex moves away from the tip. It may stay at a constant distance (e.g. fig. 3(e)), and we end up in a NESS called vortex-antivortex state (VAVS). If the antivortex moves out of the system, we again get a VS (fig. 3(f)).

The two transitions lead to very different paths in configuration space. The reason why two different barriers have to be passed, as well as why one barrier is higher than the other, can be found in the topology of the system: For the transition $\mathrm{CS} \rightarrow \mathrm{VS}$, a vortex must be created. However, an isolated vortex cannot be created, as it represents a topological defect, which violates vorticity conservation (the total number of vortex and antivortex cores). Only VAVPs can be created, and thus the energy barrier here is higher than that of the $\mathrm{VS} \rightarrow \mathrm{CS}$ transition. The reason why the latter transition (which violates vorticity conservation) can occur at all, is that here the antivortex core interacts with the open system boundary. The different transition paths of the PS lead to a hysteresis.

Influence of domain walls. - In the section above we studied a perfect system in order to get an insight into the occurring NESSs. However, a real magnetic specimen contains domain walls, which the tip has to pass through. The open question is, how stable the above characterised 

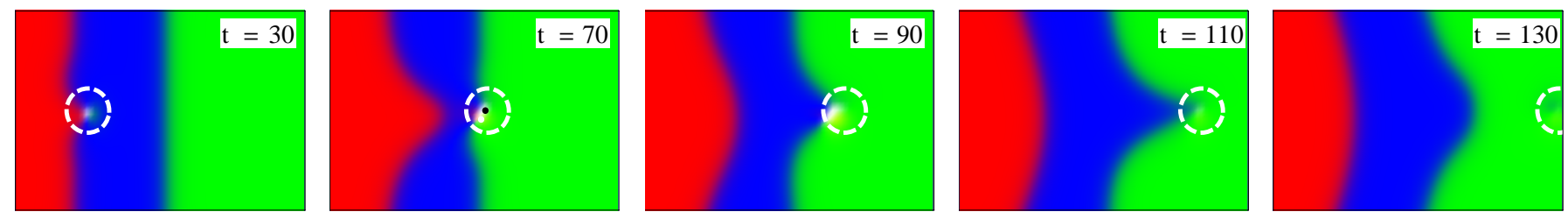

Fig. 5: (As a movie and colour online) Fast magnetic tip (marked by the dashed circle, $w=3, v=0.5$ ), interacting with two domain walls, with the domain wall distance $\mathcal{W}=20$. The snapshots show a part of the whole system containing $50^{2}$ spins. The high excitation may lead to the creation of a VAVP, see (c). As the NESS of the system is a CS, the VAVP is annihilated again, leaving the initial domain wall configuration.
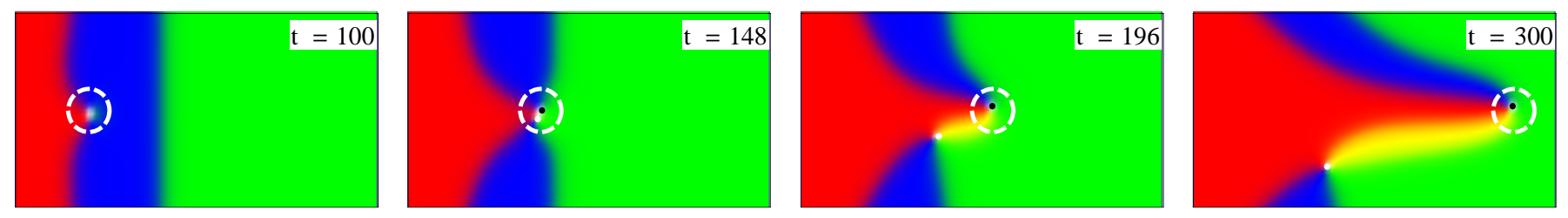

Fig. 6: (As a movie and colour online) Slow, but strong magnetic tip ( $w=5, v=0.3$ ), interacting with two domain walls. Again only a part of the system $(70 \times 50$ spins $)$ is plotted. A VAVP is created. As the NESS for the present $v-w$ combination is the VS, the vortex stays bound by the tip, and the antivortex travels out of the system, leaving a VS instead of the domain wall state.
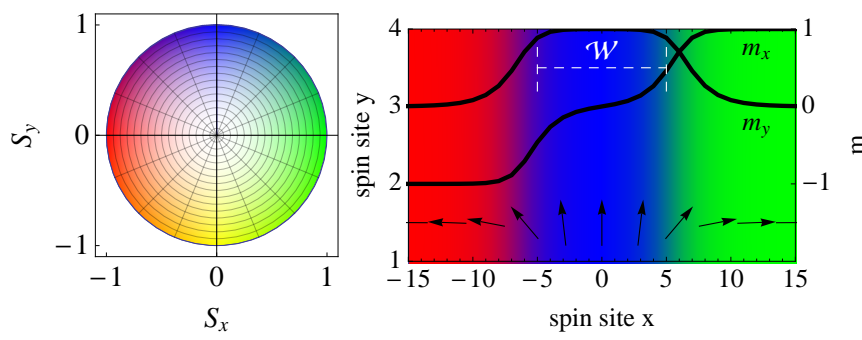

Fig. 4: (Colour on-line) Legend for the colour coded plots, and the equilibrium configuration of a system with two domain walls, as it is used for the simulations as an initial state. The width of the middle domain $\mathcal{W}$ is a free parameter.

NESSs are against e.g. domain walls. In order to answer this question we now initialise a system which contains two $\pi / 2$ domain walls in equilibrium, where the distance between the two domain walls (or the length of the middle domain) is a free parameter $\mathcal{W}$ ( $c f$. fig. 4). Additionally we fix the boundaries in $y$-direction by the equilibrium magnetisation, as it is sketched in fig. 4, to emulate infinitely long domain walls. The initial condition corresponds to the simulations initialised in the CS above, as we always start in the left domain. Let us start with a weak excitation $(w \rightarrow 0)$. Then the domain wall configuration is not disturbed by the scanning tip. We may observe a slight bending when the tip just passes by (like in the very left panel of fig. 5), which soon relaxes again, like a rubber band. After the tip has passed the domain walls the configuration is again in its initial state.

When the perturbation by the tip is stronger, a VAVP may nucleate at the domain walls, even if the energy of the moving tip alone is not sufficient, as the domain walls provide additional energy ( $c f$. fig. 5). Which state is finally adopted depends on the NESS. If the corresponding state is the CS, the VAVP get annihilated, and the initial domain wall state appears again. If the corresponding state is the VS, the distance between the vortex and the antivortex increases. Finally, the antivortex stays at the system boundary (as we have fixed boundary conditions in this part the antivortex cannot leave the system), leaving a VS that trails along with the tip. We observed that in the coexistence regime (CS \& VS) the system ends up in the VS. The PS is not influenced by the domain wall. It may occur that at the domain walls additional VAVPs nucleate, which after a short lifetime annihilate again.

The influence of the domain wall distance $\mathcal{W}$ is the following: The smaller $\mathcal{W}$, the larger is the energy density, the domain wall may provide to a VAVP creation process. Accordingly, the lowest tip strength $w$, at which a VAVP is created, is larger for larger $\mathcal{W}$.

Finally we discuss the influence of the boundary conditions. For instance from the right panel of fig. 6 one may claim that the fixed boundaries have a strong impact on the stability of the VS, and enforce the annihilation of the antivortex, as the separation of the VAVP generates continuously growing domain walls. These may become arbitrarily large and thus energetically much more unfavorable than the domain wall state. To get an annihilation process, vortex and antivortex must first move toward each other. This is only possible when the vortex is released from the tip first, as a free moving vortex or antivortex cannot move at velocities comparable to that of the tip. If the vortex is released or not from the tip depends solely on the current NESS, and not e.g. on the tailing domain walls, as they cannot provide additional energy density un- 

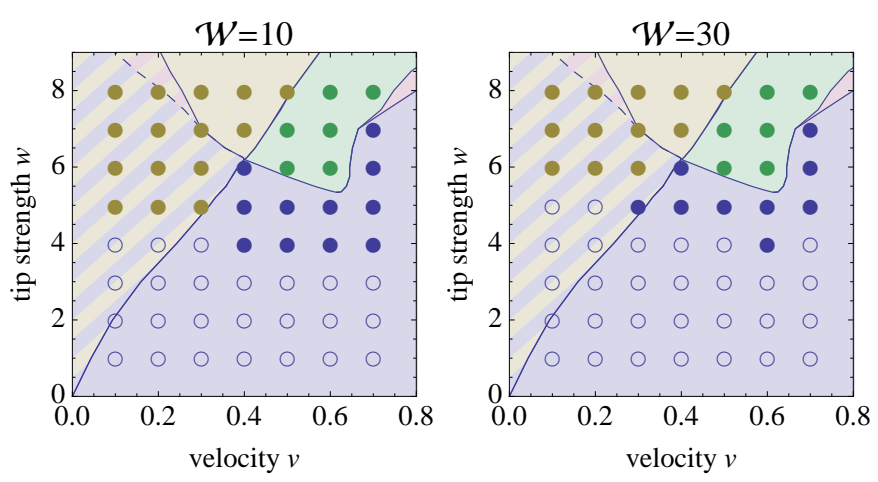

Fig. 7: (Colour on-line) Influence of domain walls on the NESS. Empty circles represent simulations, where the system does not create any VAVP, filled circles represent these simulations where at least one VAVP is created. The colour coding denotes which final state has been observed: Blue circles represent the $\mathrm{CS}$, yellow ones the VS, and green ones the PS.

der the tip for a vortex release event. All observed VAVP annihilation processes occur directly after the tip-domain wall interaction, where the events under the tip may be seen as completely decoupled from the walls.

Conclusion. - A tip, which is aligned above a ferromagnet with an easy plane anisotropy, and magnetised perpendicular to the easy plane, energetically stabilises a vortex state in the substrate. The vortex state is stable against slight perturbations, occurring e.g. when the tip is moved with constant velocity $v$ parallel to the substrate. At a threshold velocity, which depends on the tip magnetisation, the substrate may perform a transition from the vortex phase to the collinear phase and vice versa, but it may also exhibit a periodic switching for strong excitations. In a real system, $w$ is replaced by an effective value, which varies with the characteristics of the tip and the distance between the tip and the substrate, because both parameters determine the field acting at the surface and thus the energy injected into the system. In summary we have three possible states the system may adopt (the CS state and the VS state with up or down polarity), and which may be switched by a moving tip. The presence of a domain wall effectively shifts the state separation line between the coexistence state and the pure vortex state to lower tip magnetisation values.

$$
* * *
$$

We thank Sebastian Angst for valuable discussions. This work was supported by the German Research Foundation (DFG) through SFB 616 "Energy Dissipation at Surfaces". A part of the work has been performed during a stay in Porto Alegre, Brazil, granted by the German Exchange Association (DAAD) through the Project Related Exchange Brazil-Germany (PROBRAL). Computing time by the Neumann Institute for Computing (NIC) is gratefully acknowledged.

\section{REFERENCES}

[1] Belavin A. A. and Polyakov A. M., JETP Lett., 22 (1975) 245.

[2] Nikiforov A. V. and Sonin E. B., Sov. Phys. JETP, 58 (1983) 373.

[3] Huber D. L., Phys. Rev. B, 26 (1982) 3758.

[4] Gouvéa M., Wysin G., Bishop A. and Mertens F., Phys. Rev. B, 39 (1989) 11840.

[5] Shinjo T., Science, 289 (2000) 930.

[6] Wachowiak A., Wiebe J., Bode M., Pietzsch O., Morgenstern M. and Wiesendanger R., Science, 298 (2002) 577.

[7] Costa B. V., Braz. J. Phys., 41 (2011) 94.

[8] Xiao Q. F., Rudge J., Choi B. C., Hong Y. K. and Donohoe G., Appl. Phys. Lett., 89 (2006) 262507.

[9] Hertel R., Gliga S., Fähnle M. and Schneider C., Phys. Rev. Lett., 98 (2007) .

[10] Van Waeyenberge B., Puzic A., Stoll H., Chou K. W., Tyliszczak T., Hertel R., Fähnle M., Brǘkl H., Rott K., Reiss G., Neudecker I., Weiss D., BACK C. H. and Schütz G., Nature, 444 (2006) 461.

[11] Yamada K., Kasai S., Nakatani Y., Kobayashi K., Kohno H., Thiaville A. and Ono T., Nature Mater., 6 (2007) 269.

[12] Mamin H. J., Rugar D., Stern J. E., Fontana R. E. and Kasiraj P., Appl. Phys. Lett., 55 (1989) 318.

[13] Lee I., Kim J., Obukhov Y., Banerjee P., Xiang G., Pelekhov D. V., Hauser A., Yang F. and Chris HamMel P., Appl. Phys. Lett., 99 (2011) 162514.

[14] Imre A., Csaba G., Metlushko V., Bernstein G. and Porod W., Physica E, 19 (2003) 240.

[15] Yamaoka T., Watanabe K., Shirakawabe Y., Chinone K., Saitoh E. and Miyajima H., Jpn. J. Appl. Phys., 45 (2006) 2230.

[16] Auslaender O. M., Luan L., Straver E. W. J., Hoffman J. E., Koshnick N. C., Zeldov E., Bonn D. A., Liang R., Hardy W. N. and Moler K. A., Nature Phys., 5 (2008) 35.

[17] Brandt E., Mikitik G. and Zeldov E., Physica $C, 470$ (2010) 782.

[18] Magiera M. P., Brendel L., Wolf D. E. and NowaK U., Europhys. Lett., 87 (2009) 26002.

[19] Magiera M. P., Brendel L., Wolf D. E. and NowaK U., Europhys. Lett., 95 (2011) 17010.

[20] Magiera M. P., Angst S., Hucht A. and Wolf D. E., Phys. Rev. B, 84 (2011) 212301.

[21] Mermin N. D. and Wagner H., Phys. Rev. Lett., 17 (1966) 1133.

[22] Häberle T., Haering F., Pfeifer H., Han L., KuerBanjiang B., Wiedwald U., Herr U. and Koslowski B., New J. Phys., 14 (2012) 043044.

[23] Thiaville A., Belliard L., Majer D., Zeldov E. and Miltat J., J. Appl. Phys., 82 (1997) 3182.

[24] Hug H. J., Stiefel B., van Schendel P. J. A., Moser A., Hofer R., Martin S., Guntherodt H.J., Porthun S., Abelmann L., Lodder J. C., Bochi G. and OHandley R. C., J. Appl. Phys., 83 (1998) 5609.

[25] García J. M., Thiaville A., Miltat J., Kirk K. J., Chapman J. N. and Alouges F., Appl. Phys. Lett., 79 (2001) 656.

[26] Landau L. D. and Lifshitz E. M., Phys. Z. Sowj., 8 
M. P. Magiera et al.

(1935) 153.

[27] Gilbert T. L., IEEE Trans. Magn., 40 (2004) 3443.

[28] García-Palacios J. and Lázaro F., Phys. Rev. B, 58 (1998) 14937. 